\title{
MODELAGEM DE UM ATERRAMENTO ELÉTRICO CONSIDERANDO SOLICITAÇÕES EM ALTA FREQUÊNCIA
}

Thiago Costa Frade* - thiagocfrade2012@gmail.com

Gustavo Lobato Campos*-gustavo.lobato@ifmg.edu.br

Natália Rodrigues de Melo*_natalia.melo@ifmg.edu.br

*Instituto Federal de Minas Gerais, IFMG - Campus Formiga

Rua Padre Alberico, 440 - Bairro São Luiz

35570-010 - Formiga - Minas Gerais, $M G$

Resumo: A proteção de sistemas elétricos e a segurança dos seres vivos em suas proximidades estão diretamente relacionadas com um confiável projeto de aterramento elétrico. O comportamento de um aterramento para baixas frequências é bem definido na literatura e possui cálculos relativamente simples, porém quando se trata de um regime transitório solicitado por descargas atmosféricas, os cálculos são complexos, além de exigir um certo esforço computacional. Desta forma, este artigo tem o objetivo de apresentar a modelagem de um aterramento solicitado por fenômenos de alta frequência, como é o caso das descargas atmosféricas. As análises são realizadas considerando a impedância de um aterramento composto por um eletrodo horizontal. A representação do aterramento é feita utilizando o modelo de linhas de transmissão. Em alguns casos, considera-se os parâmetros elétricos do solo constantes e, em outros, variáveis com a frequência. Destaca-se que este trabalho apresenta aplicação prática de disciplinas como Eletromagnetismo e Algoritmos e Programação, sendo assim uma importante ferramenta para despertar o interesse dos discentes, por meio da metodologia de aprendizagem ativa.

Palavras-chave: Aterramentos elétricos. Descargas atmosféricas. Modelagem. Aprendizagem ativa.

\section{INTRODUÇÃO}

Os estudos referentes ao tema aterramentos elétricos têm se tornado cada vez mais frequentes devido à importância deste elemento para o funcionamento adequado do sistema elétrico. Os sistemas de aterramento apresentam uma resposta em regime permanente e outra em regime transitório. O regime permanente corresponde às respostas em baixas frequências, como por exemplo, um curto circuito e correntes de longa duração. A resposta transitória está associada a altas frequências, como é o caso das ondas impulsivas de descargas atmosféricas (SILVA, 2016).

O comportamento do aterramento elétrico, considerando fenômenos solicitantes de baixa frequência, é bem definido na literatura e os cálculos para determinação do comportamento do aterramento são relativamente simples (IEEE, 2013). Em contrapartida, quando o aterramento é submetido a correntes impulsivas, como é o caso das descargas atmosféricas, o seu comportamento se difere do observado para fenômenos de baixa frequência e há uma 
complexidade maior na modelagem dos fenômenos eletromagnéticos associados às correntes impulsivas (VISACRO, 2007).

A utilização de modelos matemáticos que forneçam uma representação física consistente de um sistema de aterramento consiste em uma alternativa adequada para investigar o seu comportamento quando solicitado por descargas atmosféricas. Por meio da modelagem é possível reproduzir as principais características de sistemas de aterramentos reais e determinar o seu desempenho. O correto equacionamento permite determinar parâmetros como sobretensões em diversos pontos do aterramento, campo eletromagnético nas proximidades do sistema aterrado, tensões induzidas em estruturas e equipamentos, tensões de passo e toque, dentre outros (ALIPIO, 2008).

A compreensão do comportamento transitório de sistemas de aterramentos solicitados por descargas atmosféricas possibilita o entendimento de conceitos fundamentais para projetos de aterramentos, que estão diretamente relacionados ao desempenho do sistema aterrado e a segurança dos seres vivos que se encontram nas suas proximidades (SUNDE, 1949; GRCEV, 1996; VISACRO, 2002). Este favorece a realização de projetos de aterramentos confiáveis, cujo objetivo principal é evitar prejuízos financeiros, associados a danos em equipamentos e, principalmente, garantir a segurança dos que se encontram nas regiões atingidas (SCHROEDER et al., 2012).

A modelagem de aterramentos possibilita a avaliação do seu comportamento frente a solicitações diversas, o que contribui para o correto dimensionamento do sistema. Por essa razão, é de fundamental importância que os estudos conduzidos nesta temática forneçam resultados confiáveis.

Neste artigo, apresentam-se os resultados obtidos por meio do modelo baseado na teoria de linhas de transmissão (TLM, transmission-line modeling model) de um aterramento composto por um eletrodo horizontal. Esse método baseia-se nas equações de propagação de ondas e possui equacionamento simples, resultando em um esforço computacional menor do que o obtido para outros métodos. Destaca-se que o desenvolvimento do mesmo tem por base a metodologia de aprendizagem ativa, onde um problema real da área foi apresentado aos discentes para prover a solução.

\section{APRENDIZAGEM ATIVA E MULTIDISCIPLINARIDADE}

Como citado este trabalho apresenta análises e resultados baseados no método de aprendizagem ativa, aplicado na aquisição dos conhecimentos necessários para o desenvolvimento da modelagem de aterramentos elétricos e assim da solução para o referido problema. Destaca-se engajamento f na busca por referenciais teóricos sobre os métodos de modelagem para aterramentos elétricos, assim como cálculos necessários para desenvolvimento de rotinas no software MATLAB. O intuito de aplicação deste método de aprendizagem é deixar discentes mais autodidatas, capacitando-os para o mercado de trabalho.

As metodologias de aprendizagem ativa permitem a integração das tecnologias para a potencialização do aprendizado, já que este poderá ocorrer em espaços e momentos fora da sala de aula articulando e exigindo vários tipos de ações e competências, além do fortalecimento de habilidades de pesquisa e alfabetização científica (SANTOS; FIGUEIREDO, 2018).

Neste contexto, o referente trabalho apresenta uma possível aplicação prática que um engenheiro, por exemplo, eletricista encontraria em campo. Observa-se que para solução do mesmo várias ferramentas, ou base em distintas disciplinas faz-se necessário. Pode-se citar, por exemplo, Eletromagnetismo, Aterramentos Elétricos, Algoritmos e Programação. Destaca-se que novas metodologias de ensino, associadas principalmente com a inter e 
multidisciplinaridade tem cada vez mais sido empregada para melhor preparar os discentes para ingressarem como profissionais na sociedade, visto que diferentes áreas de estudo podem atuar em conjunto.

\section{DESENVOLVIMENTO DO MODELO}

O modelo TLM considera que um eletrodo pode ser dividido em $\mathrm{M}$ segmentos, sendo que cada segmento é representado por um circuito composto por indutância (L), capacitância (C) e condutância (G). Nesse caso, a resistência por metro é desconsiderada pois as perdas internas ao condutor devido à resistência são pequenas quando comparadas com as perdas para o solo. A Figura 1 representa o circuito equivalente de um eletrodo utilizando o modelo TLM.

Figura 1 - Circuito elétrico simplificado de um eletrodo utilizando o modelo TLM.
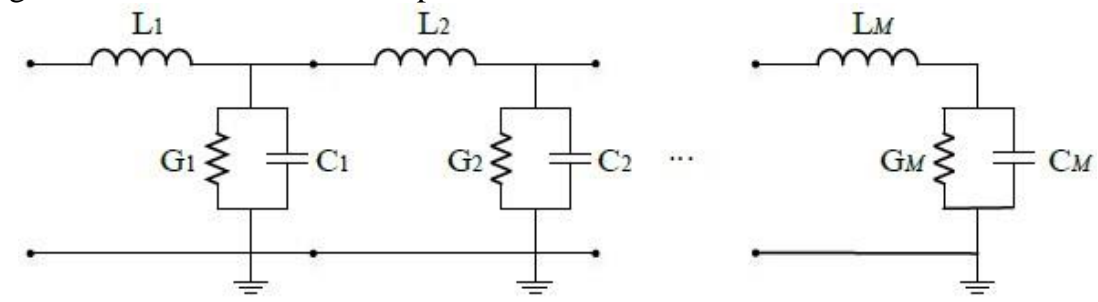

Fonte: Silva e Alves (2016).

\subsection{Cálculo dos parâmetros do solo}

Para o cálculo da permissividade do solo, considerando os parâmetros do solo invariáveis com a frequência, tem-se a Equação (1):

$$
\varepsilon=\varepsilon_{r} \varepsilon_{0}
$$

Sendo $\varepsilon$ a permissividade do solo, $\varepsilon_{\mathrm{r}}$ a permissividade relativa do solo e $\varepsilon_{0}$ a permissividade do vácuo (aproximadamente $8,854 \times 10^{-12} \mathrm{~F} / \mathrm{m}$ ).

As expressões que permitem calcular os parâmetros elétricos do solo considerando a variação dos mesmos com a frequência, foram obtidas com base nas medições de campo conduzidas por Visacro et al. (2011). Para cálculo da condutividade $(\sigma)$ e permissividade relativa do solo, respectivamente tem-se as Equações (2) e (3), sendo as mesmas determinadas por Alípio e Visacro (2014):

$$
\begin{gathered}
\sigma=\sigma_{0}+\sigma_{0} h\left(\sigma_{0}\right)\left(\frac{f}{1 M H z}\right)^{\gamma} \\
\varepsilon_{r}=\frac{\varepsilon_{\infty}^{\prime}}{\varepsilon_{0}}+\frac{\tan (\pi \gamma / 2) 10^{-3}}{2 \pi \varepsilon_{0}(1 M H z)^{\gamma}} \sigma_{0} h\left(\sigma_{0}\right) f^{\gamma-1}
\end{gathered}
$$

Em que $\sigma_{0}$ é a condutividade do solo em baixas frequências $(100 \mathrm{~Hz}) \varepsilon^{\prime} / \varepsilon_{\mathrm{r}}$ é a permissividade relativa em altas frequências e fé a frequência.

Os parâmetros $h\left(\sigma_{0}\right), \gamma \varepsilon^{\prime}{ }_{\infty} / \varepsilon_{\mathrm{r}}$ estão indicados na Tabela 1 e podem ser escolhidos de modo a se obter resultados medianos, relativamente conservativos e conservativos. Vale ressaltar que para os resultados que serão apresentados foram considerados resultados medianos. 
Tabela 1 - Parâmetros do modelo proposto.

\begin{tabular}{c|c|c|c|}
\hline & Função $\mathrm{h}\left(\sigma_{0}\right)$ & $\gamma$ & $\varepsilon_{\infty}^{\prime} / \varepsilon_{\mathrm{r}}$ \\
\hline Resultados medianos & $\mathrm{h}_{1}=1,26 \mathrm{x} \sigma_{0}{ }^{-0,73}$ & 0,54 & 12 \\
\hline Resultados relativamente conservativos & $\mathrm{h}_{2}=0,95 \mathrm{x} \sigma 0_{0}^{-0,73}$ & 0,58 & 8 \\
\hline Resultados conservativos & $\mathrm{h}_{3}=0,70 \mathrm{x} \sigma_{0}^{-0,73}$ & 0,62 & 4 \\
\hline
\end{tabular}

Fonte: Alípio e Visacro (2014)

\subsection{Cálculo dos parâmetros elétricos}

Considerando um aterramento composto por um eletrodo horizontal e os parâmetros do solo invariantes com a frequência, é possível determinar os parâmetros elétricos indutância (L), condutância (G) e capacitância (C) pelas Equações (4), (5) e (6), respectivamente.

$$
\begin{gathered}
L=\frac{\mu_{0}}{2 \pi}\left[\ln \left(\frac{2 L}{r}\right)-1\right] \\
G=\frac{\pi}{\rho_{0}}\left[\ln \left(\frac{2 L}{\sqrt{2 r h}}\right)-1\right]^{-1} \\
C=\pi \varepsilon\left[\ln \left(\frac{2 L}{\sqrt{2 r h}}\right)-1\right]^{-1}
\end{gathered}
$$

Sendo, $\mu_{0}$ a permeabilidade magnética do vácuo, $\rho$ a resistividade do solo e $\varepsilon$ a permissividade elétrica. Com relação ao eletrodo, L é o comprimento, r é o raio e h é profundidade que o eletrodo está inserido no solo.

\subsection{Cálculo da impedância de aterramento}

A impedância de entrada do sistema $(Z)$, que corresponde à impedância de aterramento, é calculada pela Equação (7).

$$
Z(f)=Z_{0}(f) \operatorname{coth}(\hat{\gamma}(f) L)
$$

Em que, $Z_{0}$ é a impedância característica e $\hat{\gamma}$ é o coeficiente de propagação, dados respectivamente pelas Equações (8) e (9).

$$
\begin{gathered}
Z_{0}(f)=\sqrt{\frac{j \omega L}{G+j \omega C}} \\
\hat{\gamma}(f)=\sqrt{j \omega L(G+j \omega C)}
\end{gathered}
$$

\section{ESTUDO DE CASO}

A partir do modelo apresentado nas seções anteriores, foram implementados códigos no software MATLAB para calcular os parâmetros do solo, os parâmetros elétricos e a impedância de aterramento a fim de caracterizar o comportamento transitório de um aterramento elétrico. 
(C) COBENGE 2020

"Os desafios para formar hoje o engenheiro do amanhã"
$\mathrm{O}$ a $\mathrm{O3}$ de dezembro

\section{Evento On-line}

\subsection{Impedância de aterramento para parâmetros do solo invariáveis com a frequência}

A Figura 2 ilustra as curvas do módulo e ângulo da impedância de aterramento obtidas para um eletrodo horizontal de $30 \mathrm{~m}$ de comprimento, raio de $7 \mathrm{~mm}$, enterrado a $0,5 \mathrm{~m}$ de profundidade em um solo homogêneo de resistividade igual a $2.400 \Omega \mathrm{m}$ e permissividade relativa igual a 15 .

Figura 2 - Módulo (a) e ângulo (b) da impedância de aterramento de um eletrodo horizontal para parâmetros do solo constantes com a frequência.

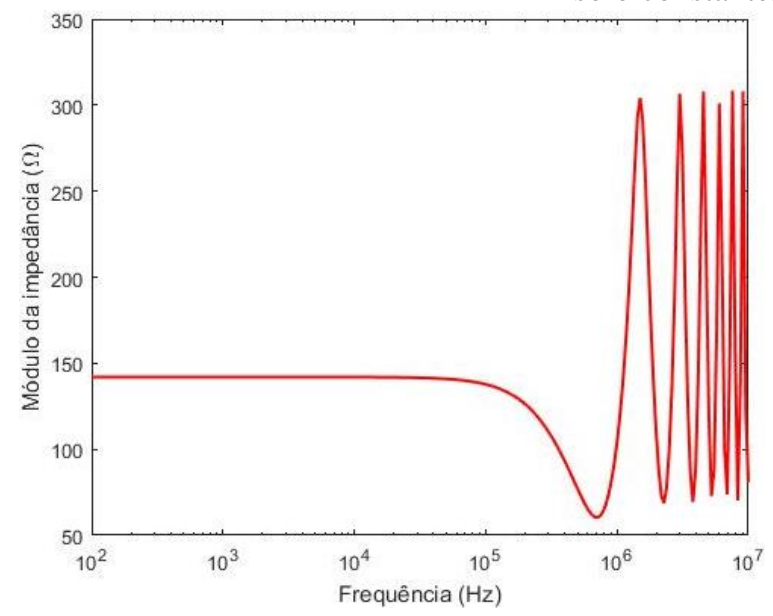

(a)

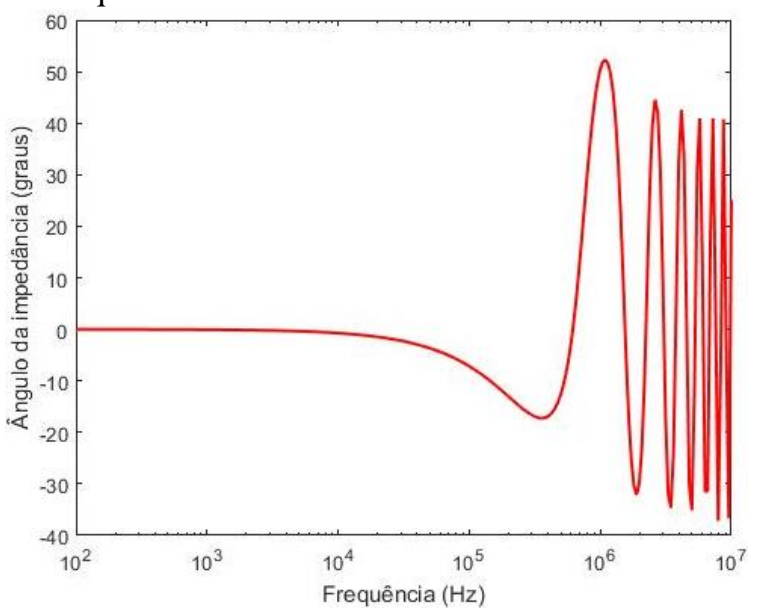

(b)

Fonte: Próprio autor.

Para validar as curvas do módulo e ângulo da impedância de aterramento da Figura 2, os resultados foram comparados com as curvas da Figura 3, obtidos por Silva e Alves (2016).

Figura 3 - Módulo (a) e ângulo (b) da impedância de aterramento de um eletrodo horizontal para parâmetros do solo variáveis com a frequência.

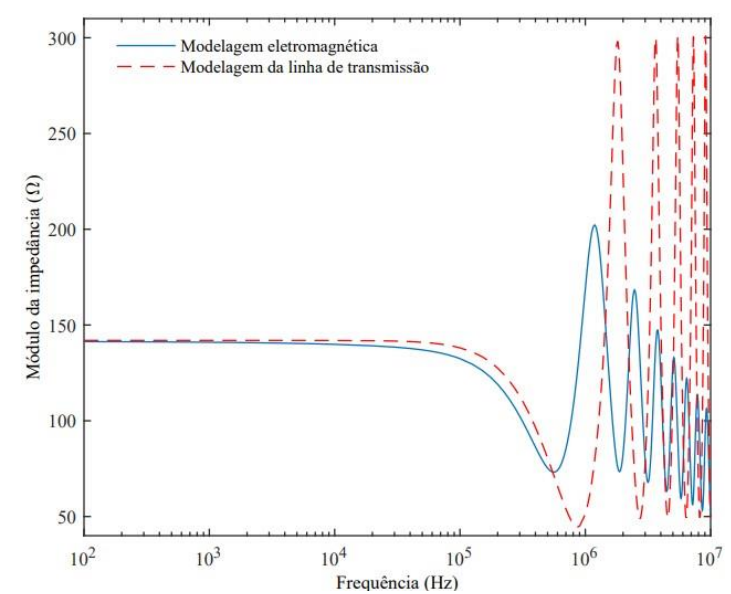

(a)

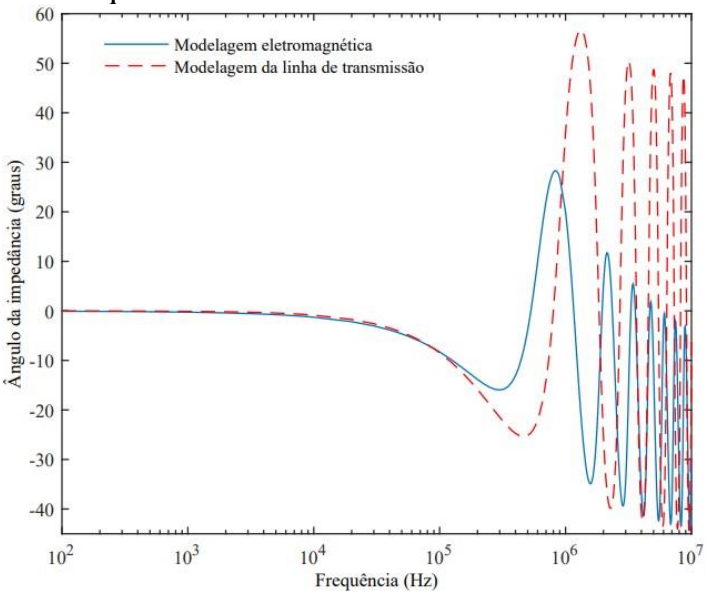

(b)

Fonte: Silva e Alves (2016)

Em baixas frequências, o módulo da impedância praticamente não varia, enquanto o ângulo é aproximadamente zero. Tal acontecimento é verificado pela reatância indutiva do 
sistema ser muito pequena e a reatância capacitiva ser muito alta, e portanto, o sistema é caracterizado apenas por uma condutância.

Para altas frequências, a impedância de aterramento apresenta características capacitivas e indutivas. Quando o ângulo possui valores negativos, predomina os efeitos capacitivos do sistema. Para ângulos com valores positivos, os efeitos indutivos prevalecem.

\subsection{Impedância de aterramento para parâmetros do solo variáveis com a frequência}

A Figura 4 representa as curvas do módulo e ângulo da impedância de aterramento para um aterramento elétrico com as mesmas características do sistema da Figura 2, porém, considerou-se os parâmetros do solo variáveis com a frequência, seguindo as equações propostas por Alípio e Visacro (2014).

É possível observar diferenças significativas entre os gráficos das Figuras 2 e 4, isso porque a permissividade elétrica do meio diminui em altas frequências, e a condutividade do solo aumenta com a frequência. Assim, o efeito global da variação desses parâmetros ocasionam a diminuição da impedância de aterramento.

Figura 4 - Módulo (a) e ângulo (b) da impedância de aterramento de um eletrodo horizontal para parâmetros do solo variáveis com a frequência.

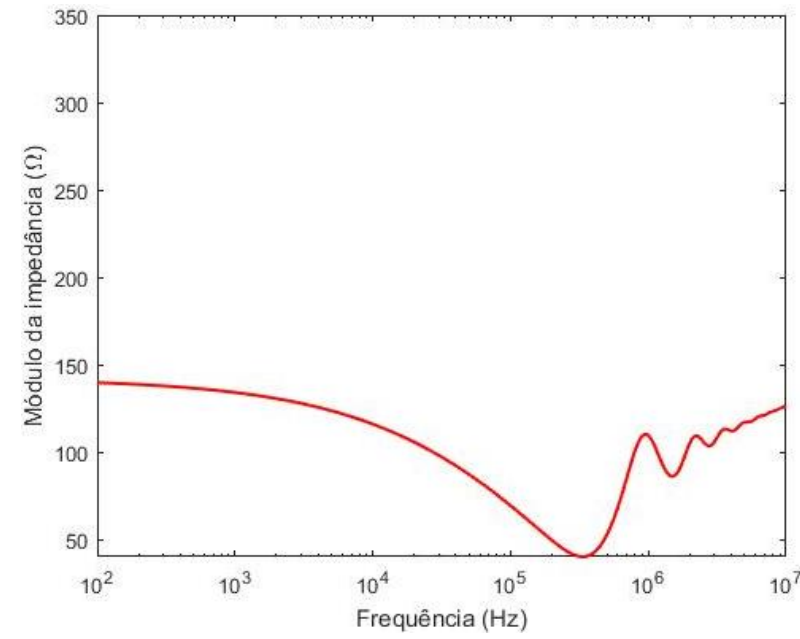

(a)

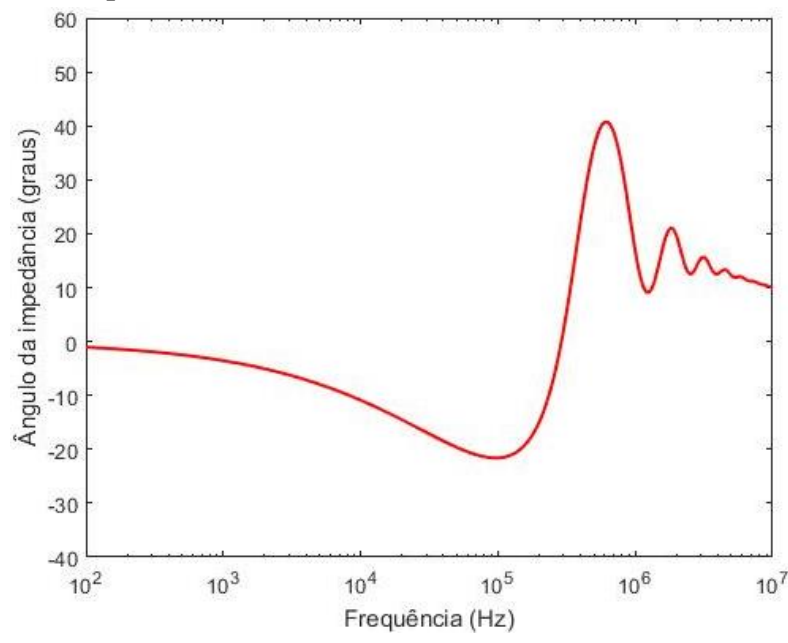

(b)

Fonte: Próprio autor.

\section{CONSIDERAÇÕES FINAIS}

Neste artigo foi apresentada modelagem baseada na teoria de linhas de transmissão de um aterramento elétrico solicitado por descargas atmosféricas. Os parâmetros elétricos do solo foram considerados constantes e variáveis com a frequência.

Observou-se que quando os parâmetros elétricos do solo são considerados constantes com a frequência, a impedância de aterramento é superestimada. Porém, ao considerar a variação dos parâmetros do solo com a frequência tem-se resultados mais próximos da realidade. Destaca-se ainda a importância do referido trabalho para apresentar conceitos e aplicações práticas vinculadas à disciplinas como Algoritmos e Programação assim como Eletromagnetismo, sendo o mesmo desenvolvido conforme conceitos da metodologia de aprendizagem ativa. Sendo esta uma das metodologias mais empregadas recentemente com objetivo não somente de apresentar situações mais próximas às reais aos estudantes, mas também orientá-los no processo de "aprender a resolver problemas por si", fato que irão 
vivenciar em sua carreira profissional, além de atrair a atenção dos discentes com ações complementares às metodologias tradicionais de ensino.

Por fim, vale ressaltar que a aplicação desta metodologia apresenta uma visão aplicada da área de Eletromagnetismo, um pouco diferente da teoria estudada em sala de aula, não só preparando o aluno para o mercado de trabalho, como também aproximando-o desta área que possui altas taxas de rejeição por partes de alunos da graduação.

\section{Agradecimentos}

Os autores agradecem ao IFMG - Campus Formiga, em especial ao GSE - Grupo de Soluções em Engenharia, pela interação e colaboração no desenvolvimento do presente trabalho.

\section{REFERÊNCIAS}

Alípio, R. e Visacro, S. (2014). Modeling the frequency dependence of electrical parameters of soil. IEEE Transactions on Electromagnetic Compatibility, 56(5):1163-1171.

Alípio, R. S. (2008). Modelagem eletromagnética de aterramentos elétricos nos domínios do tempo e da frequência. Dissertação de Mestrado, Centro Federal de Educação Tecnológica de Minas Gerais-CEFET/MG.

Grcev, L. D. (1996). Computer analysis of transient voltages in large grounding systems. IEEE

Transactions on Power Delivery, 11(2):815-823.

IEEE Std 80 (2013). IEEE Guide for Safety in AC Substation Grounding. Substations Committee of the IEEE Power Engineering Society.

MATLAB (2020). The Mathworks, Incorporation, USA. Disponível em: <https://www.mathworks.com>. Acesso em: 22 de abr. de 2020.

Schroeder, M. A., Afonso, M. M., Oliveira, T. A., \& Assis, S. C. (2012). Computer analysis of electromagnetic transients in grounding systems considering variation of soil parameters with frequency. Journal of Electromagnetic Analysis and Applications, 4(12), 475.

SANTOS, Carolina Maia dos; FIGUEIREDO, Fernanda Abreu de Moraes. ESTUDOS SOBRE METODOLOGIAS DE APRENDIZAGEM ATIVA NA ENGENHARIA PUBLICADOS NOS ANAIS DO ENEGEP DE 2013 A 2017: Análise do Perfil Acadêmico e Instituições de Origem dos Autores. In: ENCONTRO NACIONAL DE ENGENHARIA DE PRODUÇÃO, 38, 2018, Maceió - AL. Artigo em Anais de Congresso. Maceió - AL, 2018. p. $1-18$.

Silva, B. P. (2016). Modelagens e solução de aterramentos sujeitos a surtos de corrente: respostas nos domínios da frequência e do tempo. Dissertação de mestrado. Universidade Federal de Goiás.

SILVA, Bárbara Pereira; ALVES, Antônio César Baleeiro. Aterramentos sob Altas Frequências: modelagem eletromagnética e por linha de transmissão: Modelagem Eletromagnética e por Linha de Transmissão. In: SIMPÓSIO BRASILEIRO DE SISTEMAS ELÉTRICOS, 6, 2016, Goiânia - GO. Artigo em Anais de Congresso. Natal-RN, 2016. p.1-7. 
Sunde, E. D. (1949). Earth conduction effects in transmission systems. Dover Publications Inc.

Visacro, S. (2007). A comprehensive approach to the grounding response to lightning currents.

IEEE Transactions on Power Delivery, 22(1):381-386.

Visacro, S., Alípio, R., Vale, M. H. M., e Pereira, C. (2011). The response of grounding electrodes to lightning currents: The effect of frequency-dependent soil resistivity and permittivity.

Visacro, S. (2002). Aterramentos elétricos: conceitos básicos, técnicas de medição e instrumentação, filosofias de aterramento. Artliber.

\title{
THE RESPONSE OF A GROUNDING SYSTEM CONSIDERING HIGH FREQUENCIES
}

\begin{abstract}
The protection of electrical systems and safety of living beings located near these systems are directly related to a reliable grounding project. The grounding system behavior is well stablished considering low frequency range and includes simple calculations. However, when subjected to lightning currents the grounding system has a very particular behavior and the modeling become more complex. This work presents the response of a horizontal electrode subjected to lightning current. The transmission line model is used to represent the grounding system. Results of the application of this methodology are presented considering soil electrical parameters constant and their frequency dependence. This work presents practical application of subjects such as Electromagnetism and Algorithms and Programming. Therefore, it can be considered an important tool to arouse the interest of students.
\end{abstract}

Keywords: Grounding system. Lightning current. Modeling. 